\title{
An Expert System for Diagnosis of Blood Disorder
}

\author{
Ayangbekun, Oluwafemi $\mathrm{J}$ \\ Department of Information \\ Systems \\ University of Cape Town \\ South Africa
}

\author{
Olatunde, Ayoola I. \\ Department of Computer \\ Science \\ Crescent University Abeokuta \\ Nigeria
}

\author{
Felix O. Bankole \\ Department of Information \\ Systems \\ University of Western Cape \\ South Africa
}

\begin{abstract}
The mortality rates in medical sector have given birth to most technology that aids in treatment of diverse ailments. The commencement of technology era in the 20 century gives a great mark to the limit of unnecessary element that causes mortality. As time is one of the major elements that deal with mortality issue, this however give birth to this research as it stands out to help in reducing the time that is used in attending to a critical patient medical condition. This is to aid the doctors and other medical practioners in attending and prescribing treatment to patients. Hence, this research takes blood disorders into consideration by finding a way to solve the issue in human being. Apart from solving or diagnosing, the need to give treatment in order to make the system more useful is necessary. This then make the research to be exposed to very large information that is taking down from two hospitals which was taken as the case study of the research. The hospitals used include UCH Ibadan and FMC IdiabaAbeokuta both in Nigeria, West Africa. The information was gathered from the hematology department and the blood department of the two hospitals respectively. The information gotten was analyzed and manipulated based on the symptoms and causes of the blood diseases/disorders and then turned into rules for easy programming into the computer. Also, forward chain and backward chain approach are used to analyze the blood disorder in which gives flexibility to the doctors on knowing more about a disease or diagnosing a patient through the system.
\end{abstract}

\section{General Terms}

Flowcharts, Expert system, Blood Disorder

\section{Keywords}

Expert System (ES), Medical Expert System (MES), Blood Disorder System (BDS), Forward Chain Approach (FCA), Backward Chain Approach (BCA), Rule Base Expert System (RBES)

\section{INTRODUCTION}

The advancement of Expert System has touched some major areas especially in health. Expert system (ES) is a branch of Artificial Intelligent that emulates the decision-making ability of a human expert [1]. Expert systems are designed to solve complex problems by reasoning about knowledge, represented primarily as IF-THEN rules rather than through conventional procedural code. The desire to safe many lives through ICT has been one of the major areas to research on. Many patients have met their end or have major damages due to slow response of test. This gives many researchers to start developing various expert systems to aid medical field so as to solve this problem. Researches have shown that we have lower rate of mortality only in the ICT era. As technology is growing, work offload and quicker response are becoming what chasing after. Many cases at the hospital always require carrying out blood disorder test before any diagnosis/treatment. The proposed system has been subjected to a voluminous amount of information gotten from the hematologist (i.e the expert in this field). The information is relatively manipulated into expert system to solve humans' blood problems. Most expert system build recently has left the issue of blood diagnosis and focuses on other major area of medicine. Although there is no singular system that can take care of all medical works, in which as well, there has been nobody that can stand out to call himself an expert in all area of medicine which has always made this a little cumbersome. As it is well known that it is the work of ICT to cater for the way to bring easiness to human life and activities mostly in all area of living. Development of ICT also help in building this kind of system so as to be able to integrate it into a later system which will be able to collect a blood sample and check it, in order to know what actually happens in the blood. ICT have already being into this area, but cannot be implemented here due to a lot of resources that will be required from the developer. Hence, the implementation of the system is based mainly on a kind of simulation which does not require taking in any blood sample but can determine the defect by analyzing it through a form of a rule based expert system.

\section{BACKGROUND}

There are two basic expert systems being used from time to time in healthcare for diagnosing blood diseases and prescribing treatments. The two major systems are: MYCIN and CADUCEUS. MYCIN is an early expert system that used artificial intelligence to identify bacteria causing severe infections, such as bacteremia and meningitis, and to recommend antibiotics, with the dosage adjusted for patient's body weight. The name derived from the antibiotics themselves, as many antibiotics have the suffix "-mycin". The Mycin system was also used for the diagnosis of blood clotting diseases. The development of MYCIN started five to six years in the early 1970s at Stanford University, where it was written in LISP as a doctoral desertion of Edward Shotlife under the supervision of Bruce Buchanan, Stanley N. Cohen and others [2]. It was created in the early Dendral expert system [2].

CADUCEUS was a medical expert system finished in the mid-1980s (first begun in the 1970s- it took a long time to build the knowledge base) by Harry Pople (of the University of Pittsburgh), building on Pople's years of interviews with Dr. Jack Meyers, one of the top internal medicine diagnosticians and a professor at the University of Pittsburgh. Their motivation was an intent to improve on MYCIN - which focused on blood-borne infectious bacteria - to focus on more comprehensive issues than a narrow field like blood poisoning (though it would do it in a similar manner); instead embracing all internal medicine [3]. CADUCEUS eventually could diagnose up to one thousand different diseases. [3] 


\subsection{Literature Review}

There are different areas in medicine where an expert system has been designed and implemented to profers solution to health status stability in human. Among these diverse areas includes an expert system for Eye, Hypertension, Pregnancy, Leukemia and several other Human diseases. Sam S. Abu [4] in his research work for eye expert system, he used CLIPS language in his research thereby serving as a tunnel to the inner workings of the body [4]. Also, J Gudu [5] in his research for expert system to diagnosis and treat hypertension in pregnancy stated that the diagnostic and treatment expert system for hypertension in pregnancy has so far remained at the testing phase of its life cycle and is yet to be implemented [5]. During his research, it was stated that there is an acute shortage of specialist obstetricians in the reproductive health division which implies that there is also scarce expert knowledge on the diagnosis and treatment of hypertension in pregnancy, yet the condition continues to kill many women of reproductive age in Kenya, hence the need to develop the Medical Expert System (MES) as an expert knowledge sharing tool to be used by other medical personnel within the reproductive health division who are not specialists in diagnosis and treatment of hypertension in pregnancy [5] is of great necessity.

More so, Azar developed an expert system for diagnosing leukemia, his programs simulate the pattern of thinking and the manner of how human operates and causes the operation of expert systems to be close to operations of human or an expert [6]. Leukemia is very common and serious cancer starts in blood tissue such as the bone marrow [6]. It causes large numbers of abnormal blood cells to be produced and enter the blood. Speed is always effective in diagnosis and treatment of Leukemia and recovery of patients, but sometimes there is no access to specialists for patients. Because of this reason, designing a system with specialist knowledge, that offers the diagnosis and appropriate treatment to patients, and providing timely treatment of patients [6] was brought into the scene.

\subsection{System Architecture}

This describes the fundamental knowledge of building the system. The basic operation of the system staring from the doctor's contact to where the heamatologists supply information is describe in the following diagram (Figure 1). Here, the doctor communicate with the user interface with the aim of using the system to diagnose patients, the user interface then contact the inference engine for query processing and retrieving of information from the knowledge base or the working memory. The knowledge base on the other hand is updated by the heamatologists so as to improve the reliability of the expert system.

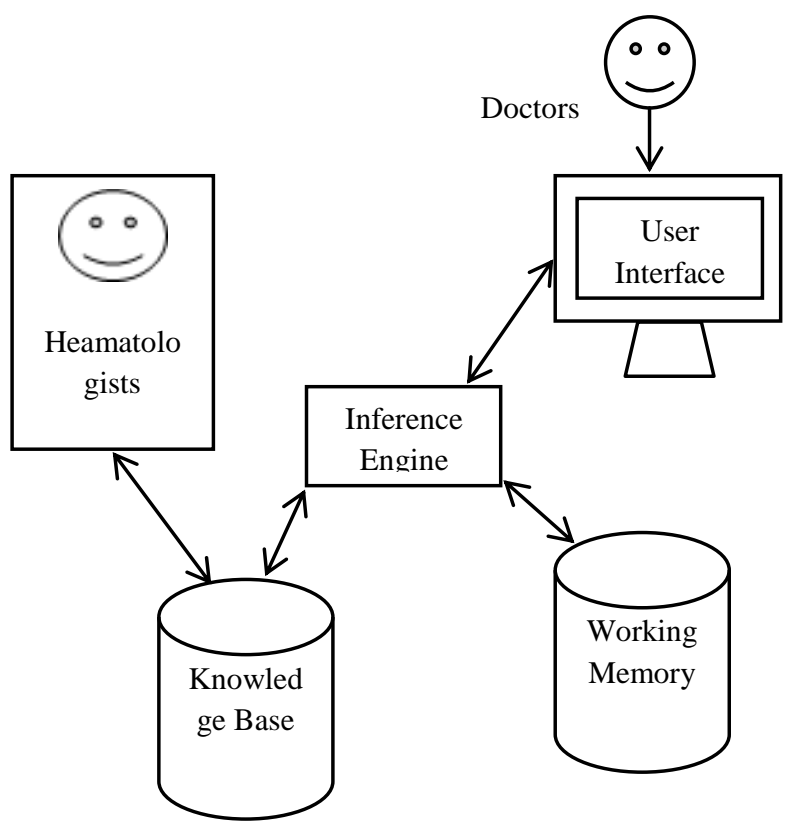

Fig 1: Expert System Architecture

\section{METHODOLOGY}

Generally to develop an expert system, a rule based method is required to analyze and compute the knowledge base. P.Santosh Kumar Patra (2010) [7] in his research used fuzzy logic to develop his expert system for the treatment of human diseases and analyze the conditions based on some natural phenomenon. Using a rule base approach such as IF, IF THEN ELSE can also give programmers the same flexibility of incorporating some natural phenomenon like that of fuzzy logic. An analysis was conducted based on data gathered on the types of blood disorders and their symptoms. The symptoms and causes of the blood disorder were taken into consideration by making the system learn. This approach allows input of all possible causes and symptoms of all the blood disorders available. After the learning process, the system can then accommodate various blood disorders. Since all the disorders are already in the database, rule based approach is then used to compare the information collected from the doctors to the one in the database in order to give accurate response.

\subsection{Research Flow}

This describes the way the whole research was conducted starting from the very start to the end. The diagram (figure 2) below shows that the research started by consultancy of the hematologists and gathering of information, and ended at the conclusion and finalizing of the whole research work by documenting and tiding up its environment. The research start with expert consultancy and move to doctor's awareness and also to data analysis follow by the rules implementation. User interface follows in the process and then the system body coding, before the final testing and conclusion. The following diagram (fig 3.5) shed light to the research flow. 


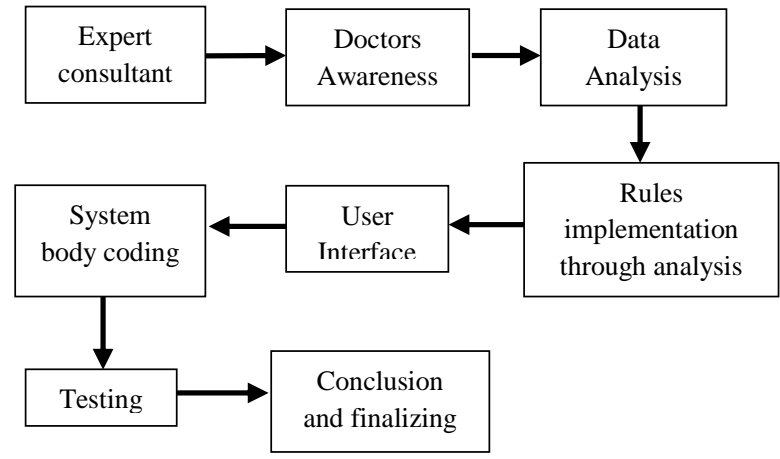

Fig 2: $\quad$ Research Flow

\section{SYSTEM DESIGN ANALYSIS}

The general flow chart of the design can only be denoted by the design flow below.

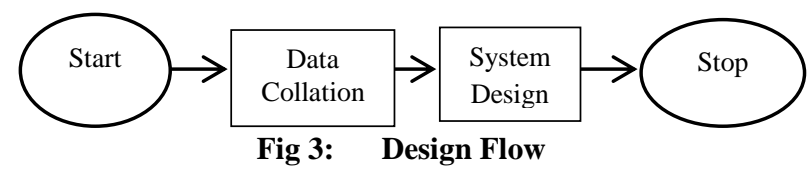

From the above diagram (figure 3), the two major concerns are the data collation and the system development. The data collation is always needed even after the developmental stages, this is done because update to already existing disorder may occur or another blood disorder may occur. This data collation is always done by the admin of the system.

\subsection{System Design Phases}

This includes all the pages and phases that make up the whole system. The system works with two basic types of users which are: Admin and Doctors. The Admin coordinate the system by giving privileges to new doctors in other to gain access to the system. The Admin also collate data from reliable sources in order to update the blood disorders that will be treated by the system. Hence, this means that the Admin is responsible to adding a new notable blood disorder based on the doctors directives and opinion. The doctors on the other hand make use of the diagnosis and treatment page in order to treat patients as described below.

\subsubsection{Login Page}

This is a pane or page where authentication is given to the users of the system. It asks for both the username and the password, so as to be able to authenticate. The same page is used for both the admin and the doctors as shown in the login flow (figure 4). The admin will need to login using his/her username and password. If they are correlated, then it will open the admin pane for the admin of the system. Else, it willcheck if the user is a valid userbased on the inbuilt MySQL database so as to allow or disallow the user from moving down to the diagnoses pane of the system.

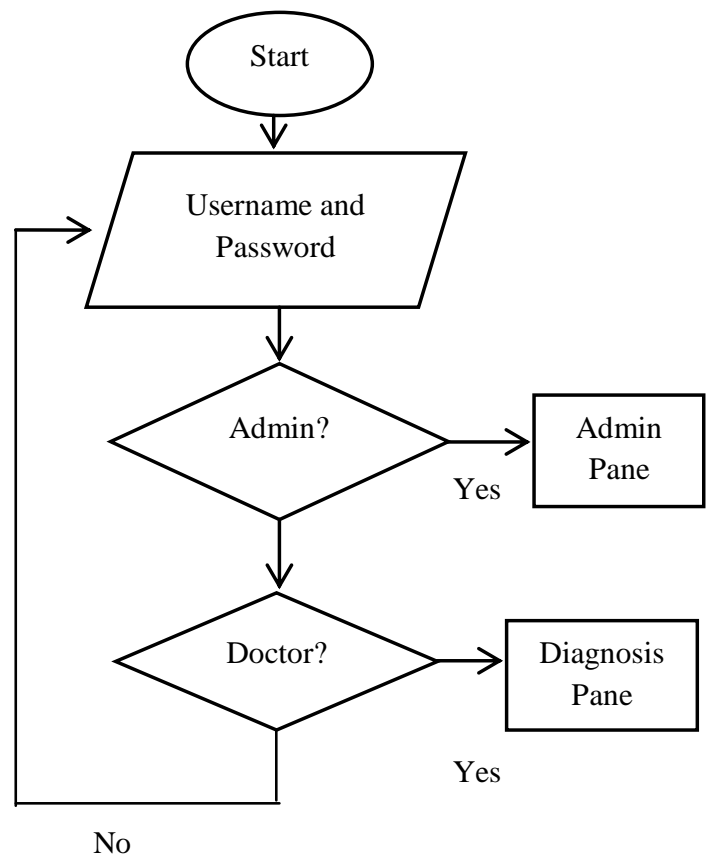

Fig 4: Login Flowchart

\subsubsection{Admin Page}

This pane is being managed only by the system administrator. Although, it is a web program where many users can login but still requires the system administrator to administer user's access. The admin pane gives privileges to the admin to create account for the users and modify their data. The admin (who understood the fall system functionality and design) has the privilege to delete and edit user's record from the database as well as adding new disorders to the system based on the experts (Doctors) instructions (figure 5).

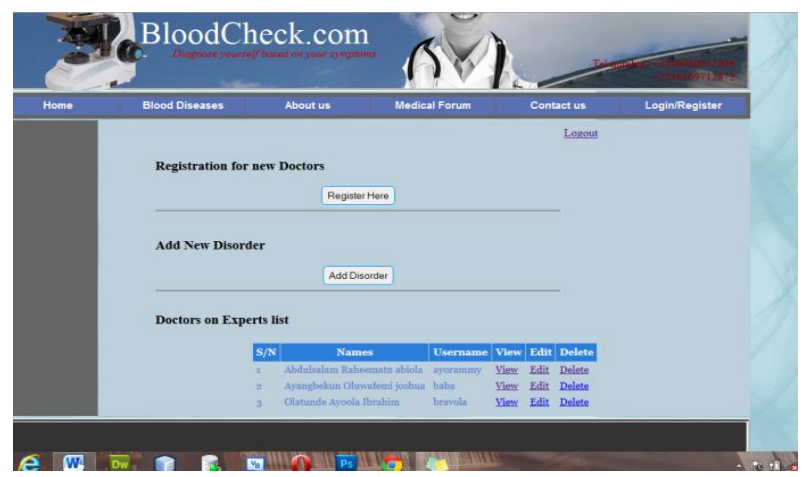

Fig 5: Admin Page

\subsubsection{Add Disorder Page}

This is the interface where new blood disorders are added to the system (figure 6) for usage by the doctors. This is done only by the admin of the system based on the experts (Doctors) recommendation/instruction because he is the only one that knows the in and out of the systems functionality. It was generally known from the information gathered that some doctors are not really conversant with the use of an expert system. It also validate against multiple entry of same disorder as shown in the add disorder flow below (figure 7). 


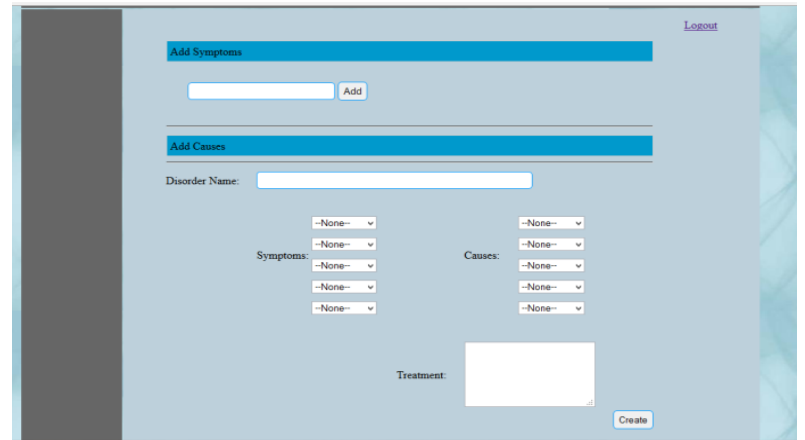

Fig 6: Add Disorder Pages
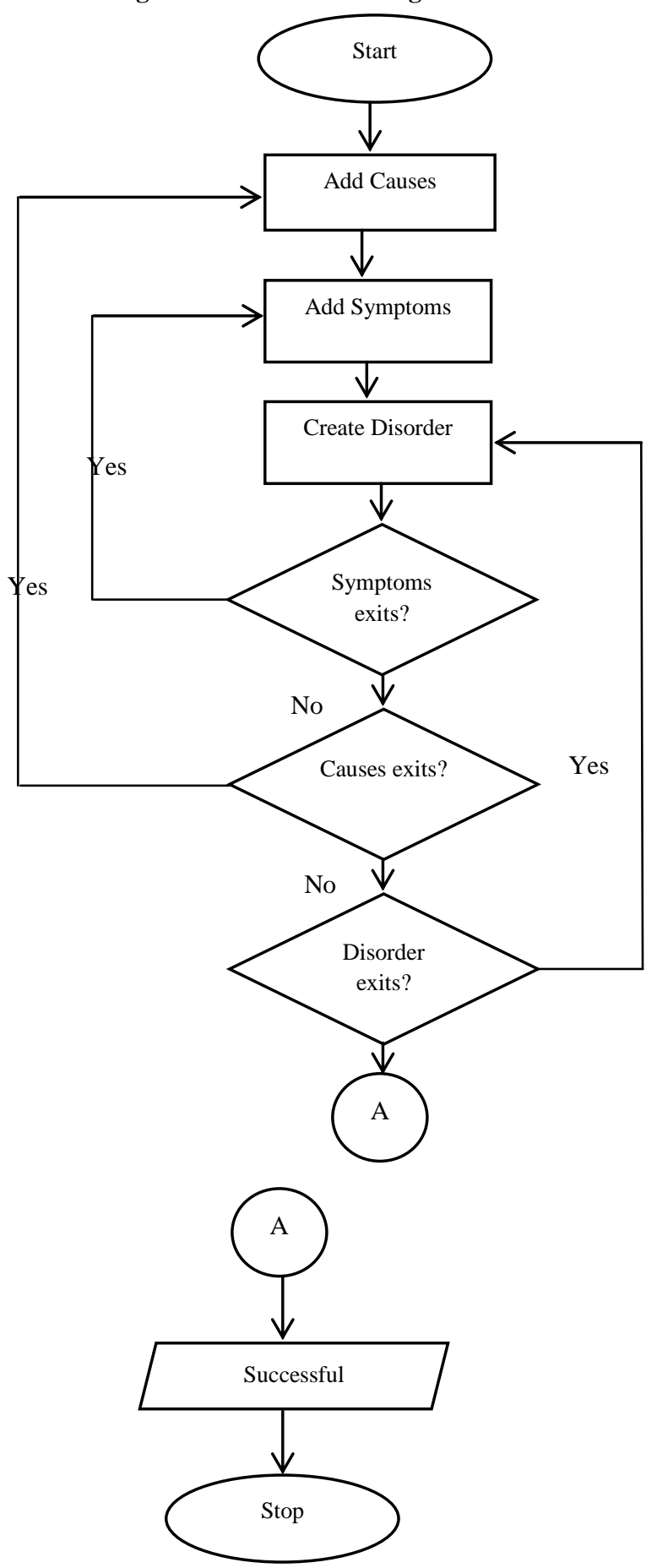

Fig 7: Add Disorder Flowchart

\subsubsection{Diagnosis Page}

This is the pane where all major research work is focused. This pane performs all diagnoses and works in two major dimensions (i.e the forward chain and backward chain dimensions). The first dimension (forward chain dimension) means getting a result based on the known facts (such as symptoms and causes). For doctors to use this feature he has already known the symptoms and causes of the patient's ailment but without knowing the actual blood disorder (figure 9). The second dimension which is the (backward chain dimensions) involves getting information about the already known blood disorder in order to aid treatment (figure 10). For doctors to use this feature, he has already known the blood disorder but do not have the relevant information about it. Hence the doctor looks for the disorder in the blood disorder list and check for the full detail (figure 8).

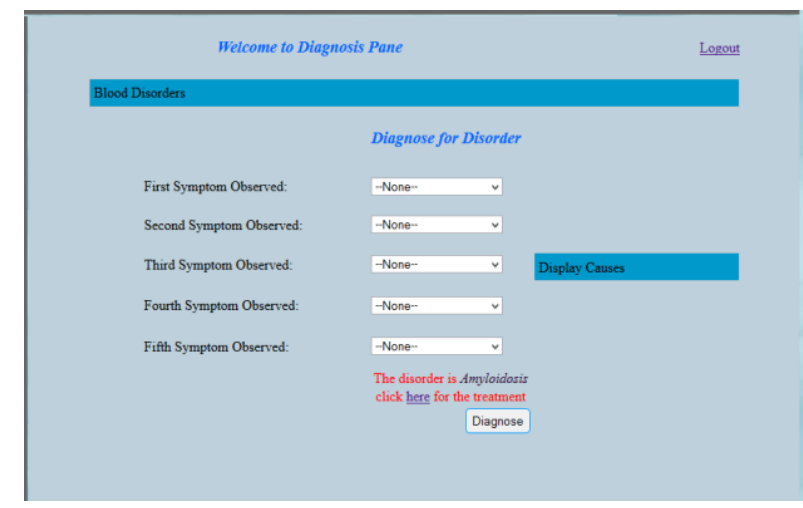

Fig 8: Diagnosis pane

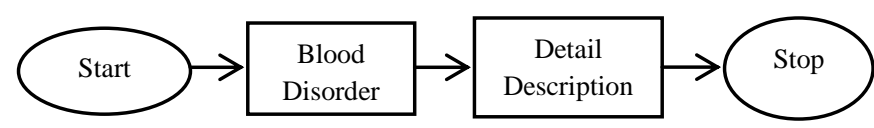

Fig 9: Forward Chain Flow

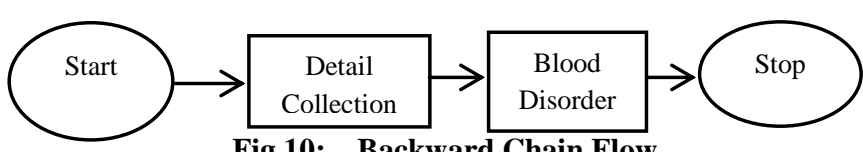

Fig 10: Backward Chain Flow

\subsubsection{Treatment Page}

This page gives full description about disorder being diagnosed. After the system identified the type of disorder based on the causes and symptoms from the diagnosis page, it then give a link (figure 8) to this page to proffers solution to the problem at hand (see figure 11). 


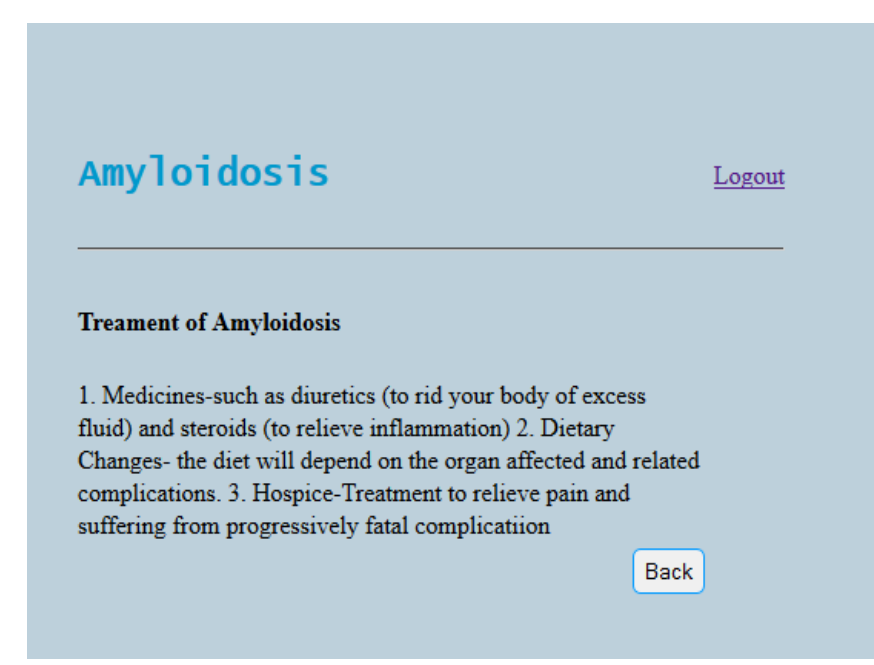

Fig 11: Treatment Page

\section{CONCLUSION}

The result of the analysis shows that the system can be used to capture diverse blood disorders. The above system goes through learning processes by adding various causes and symptoms that are likely to ever occur in any blood disorder type. This makes it easier for the system to gain knowledge about the disorders with respect to their causes and symptoms. Also, the system can be developed using other databases like
SQL which even can bring much easy access to the developmental phase depending on how it can be deploy. Hence, from the above system development, a need to add security issues is also necessary so as to guide the system from being used by an unauthorized user. Wrong usage of this system can lead to bad or adverse effect on humans live.

Moreover, this expert system has been developed as a single unit with greater flexibility to allow other medical expert on blood disorders to effectively communicate and relate with each other thereby forming a more robust system

\section{REFERENCES}

[1] Wikipedia.org/ /wiki/artificial_intelligence. (2011). Retrieved March 2014, from wikipedia.com: www.wikipedia.org

[2] Wikipedia.org/ wiki/Mycin. (2011). Retrieved March 2014, from wikipedia.com: www.wikipedia.org

[3] Wikipedia.org/wiki/Caduceus. (2011). Retrieved March 2014, from wikipedia.com: www.wikipedia.org

[4] Samy S.Abu, A. Z. (2005). Journal of Theoretical and Applied Information Technology.

[5] J. Gudu, D. G. (2012). International Journal of Bioscience, Biochemistry and Bioinformatics, 5.

[6] Azar, A. G. (2004). International Journal of Computer \& Information Technologies.

[7] P.Santosh Kumar Patra, 2010 An Expert System for Diagnosis of Human Diseases 